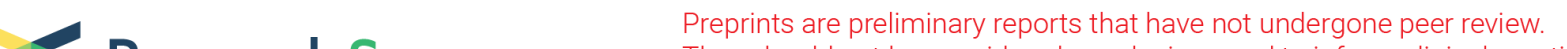 $\begin{array}{ll}\text { Research Square } & \begin{array}{l}\text { They should not be considered conclusive, used to inform clinical practice, } \\ \text { or referenced by the media as validated information. }\end{array}\end{array}$
}

\section{The Global, Regional, and National Burden of Nasopharyngeal Carcinoma and Its Attributable Risk Factors in 194 Countries and Territories, 2007- 2017}

\section{Zhisen Shen}

Department of Otorhinolaryngology Head and Neck Surgery, the Affiliated Lihuili Hospital, Ningbo University

\section{Yujie Cao}

Department of Otorhinolaryngology Head and Neck Surgery, the Affiliated Lihuili Hospital, Ningbo University

\section{Lin Luo}

Department of Otorhinolaryngology Head and Neck Surgery, the Affiliated Lihuili Hospital, Ningbo University

\section{Liyuan Han}

Hwa Mei hospital, University of Chinese Academy of Sciences

Jun Li

Department of Otorhinolaryngology Head and Neck Surgery, the Affiliated Lihuili Hospital, Ningbo University

\section{Liuqian Wang}

Department of Otorhinolaryngology Head and Neck Surgery, the Affiliated Lihuili Hospital, Ningbo University

\section{Yaqin Lu}

Department of Otorhinolaryngology Head and Neck Surgery, the Affiliated Lihuili Hospital, Ningbo University

\section{Shanshan Gu}

Department of Otorhinolaryngology Head and Neck Surgery, the Affiliated Lihuili Hospital, Ningbo University

\section{Hongxia Deng ( $\square$ xixizb@163.com )}

Department of Otorhinolaryngology Head and Neck Surgery, the Affiliated Lihuili Hospital, Ningbo University

\section{Research Article}

Keywords: Global Burden of Diseases, incidence, DALY, death, risk, Nasopharyngeal carcinoma 
Posted Date: August 24th, 2021

DOl: https://doi.org/10.21203/rs.3.rs-778985/v1

License: (c) (i) This work is licensed under a Creative Commons Attribution 4.0 International License. Read Full License 


\section{Abstract}

\section{Background}

So far, no research about the burden of the incidence rate, Disability-Adjusted Life Years (DALYs), and death rate associated with nasopharyngeal carcinoma (NPC) has been reported on the global, regional, and national levels. In this paper, we provide an overview of the most recent global epidemiology of nasopharyngeal cancer with data obtained from the Global Health Data Exchange (GHDx) repository.

\section{Methods}

We utilized the reports of the Global Burden of Diseases (GBD), Injuries, and Risk Factors Study in 2017 to estimate the burden of NPC in 194 countries and territories by age, sex, and Socio-Demographic Index (SDI) from 2007 to 2017. Moreover, we assessed the risk factors of NPC-related DALYs and deaths through the Comparative Risk Assessment Framework.

\section{Results}

In the year 2017, 10.978 million new NPC cases with a 95\% uncertainty interval of 10.444 to 11.556 million were recorded globally, whereas the DALYs and deaths were 2.09 million cases with a $95 \% \mathrm{UI} 2$ to 2.17 million and 695.5 thousand cases with $95 \%$ UI 668.7 to 722.7 thousand, respectively. From 2007 to 2017 , the estimated incidence rate of NPC decreased by $2.3 \%$ with $95 \%$ uncertainty interval $-7 \%$ to $3.28 \%$, the estimated DALY rate decreased by $4.95 \%$ with $95 \%$ uncertainty interval from $-8.46 \%$ to $-1.24 \%$, and the estimated death rate decreased by $3.01 \%$ with $95 \%$ uncertainty interval $-6.36 \%$ to $0.43 \%$. The agestandardized incidence, DALY, and death rates in 2017 were all the highest among the countries located in the middle-SDI quintiles region. At the GBD regional level, the most severe age-standardized incidence, DALY, and death rates in 2017 occurred in Oceania, followed by Southeast Asia and East Asia. From 2007 to 2017, the Caribbean and South Asia have the most increase in percentage in age-standardized incidence, DALY, and death rates. At the national level, countries like Ukraine, Jamaica, and the Dominican Republic reported the largest percentage increases in the age-standardized incidence, DALY, and death rates in a decade. From 2007 to 2017, DALYs and death rate of NPC increased by 18.99\% (95\%Ul:13.34\% $25.73 \%$ ) and $23.5 \%$ (95\%Ul:17.76\% - 29.84\%), respectively. While the estimated age-standardized attributable risk DALY rate and the age-standardized attributable risk mortality rate decreased by $5.55 \%$ (95\% UI: $-9.93 \%-0.34 \%)$ and $4.25 \%$ (95\% Ul: $-8.64 \%-0.56 \%)$.

\section{Conclusion}

The middle-SDI quintiles had the highest age-standardized incidence, DALY, and death rates in 2017. The largest increases in age-standardized incidence, DALY, and death rates were reported in the Caribbean and South Asia from 2007 to 2017, especially in countries such as Ukraine, Jamaica, Dominican Republic, and Dominica. In these regions, Alcohol consumption, Smoking, and A diet short of fruits were the primary three risk factors contributing to both DALYs and deaths in 2017. 


\section{Introduction}

Nasopharyngeal carcinoma (NPC) is a malignant epithelial tumor usually originates in the Fossa of Rossenmueller[1]. NPC is uncommon in most areas of the world but poses a significant public health burden in endemic regions[2].

Although there are periodic literatures indicating that the incidence of and mortality from NPC is declining in endemic areas[3], no detailed quantitative global, regional, or national estimates of the NPC incidence and NPC-associated disability-adjusted life years (DALYs) and deaths are available across countries and territories.In this article, the data of the Global Burden of Diseases, Injuries, and Risk Factors Study (GBD) 2017 was used to assess the global, regional, and national incidence of NPC and NPC-associated DALYs and deaths,in addition to its associated risk factors,across 194 countries and territories, for the years 2007 to 2017. Such information is of great medical significance for the prevention of NPC among highrisk groups in key geographic areas.

\section{Materials And Methods}

\section{Overview}

The general methodology of GBD 2017 has been presented elsewhere[4-7]. GBD 2017 systematically studied 359 diseases and injuries, 282 causes of death, and 84 behavioral, environmental, occupational, and metabolic risk factors. GBD 2017 is based on a geographical hierarchy consisting of 194 countries and territories grouped into 21 regions and 7 GBD super-regions. The GBD Study followed the Guidelines for Accurate and Transparent Health Estimates Reporting (GATHER) statement[8].

\section{Data sources}

Data on the burden of NPC comes from an online database, the Global Health Data Exchange (GHDx) query tool (http://ghdx.healthdata.org/gbd-results-tool), which is a continuously updated global collaboration that uses the latest epidemiological data to compare and evaluate the health loss caused by 359 diseases in 194 countries and regions.

\section{Disability-adjusted life years (DALYs)}

In this article, DALYs was used as the standard indicator to quantify the burden ${ }^{6}$ and was calculated by combining years of life lost (YLLs) and years lived with disability (YLDs). In GBD 2017, the average disability weight was multiplied by the age-, sex-, and region-specific prevalence to derive YLDs. The disability weight is used to determine the severity of the disease and ranges from 0 (completely healthy) to 1 (equivalent to death).

\section{Socio-demographic index (SDI)}

In this study, the state of socioeconomic development of the country is reflected in SDI which was modified in GBD 2017[4-6]. SDI is combined of the total fertility rate of the population under 25, average 
education level of the population over 15 years old, and lag-distributed income per capita on a scale from 0 (less developed) to 1 (most developed)[9].

\section{Risk factors}

The literature on risk factors and risk-attributable DALY and death had been reported before[10]. The assessment of exposure level and risk-attributable DALYs and deaths was estimated by the comparative risk assessment framework from 2007 to 2017. The proportions of DALYs and deaths that could be attributed to a specific risk were assessed by the counterfactual scenario of theoretical minimum risk exposure level[10].

\section{Statistical analysis}

Rate per 100,000 population was age-standardized in the light of the GBD world population[9]. The 95\% uncertainty interval (UI) was reported for estimates, in the process of cancer assessment, the calculation method of Uls were $2.5 \%$ and $97.5 \%$ of the distribution of 1000 lots, with the uncertainty disseminated through each step.

\section{Results}

\section{The incidence counts and age-standardized incidence rate per 100,000 population by countries and territories}

Table 1 shows the number of incident NPC cases for 2017, the age-standardized incidence rate per 100,000 for 2017 , the percentage change in all-age incidence counts, and the percentage change in agestandardized incidence rates between 2007 and 2017 by countries and territories. Globally, from 2007 to 2017, the number of incident NPC cases increased to 10.978 million ( $95 \%$ uncertainty interval [UI]: 10.444-11.556), representing an increase of 20.34\% (95\% Ul:14.34-27.29\%) in the number of global incident cases. The age-standardized incidence rate of NPC was 1.35 (95\% Ul:1.28-1.42) per 100,000 population in 2017, representing a decrease of 2.3\% (95\% UI:-7-3.28\%) from 2007 to 2017.

In 2017 , the middle-SDI quintiles (1.76 [1.63-1.9] per 100,000 population) had the highest agestandardized incidence rate of NPC among all seven super-regions(Table 1, Fig. 1A). The high-middle-SDI quintiles were the only quintiles to experience an increase in the percentage changein age-standardized incidence rate(0.4\% [- 10.67-14.22\%]), whereas the high-SDI quintiles showed the greatest decrease ($13.89 \%$ [ $18.35 \%$ to $-8.6 \%$ ) (Table 1, Fig. 1D). In all years from 2007 to 2017, the gender patterns between males and females were similar, although the numbers and rates of males were always higher than females (Supplementary Tables 1 and 2).

In 2017, at the geographic region level,the highest age-standardized incidence rates of NPC were observed at Oceania(3.01 [2.3-3.91] per 100,000 population), Southeast Asia (2.54 [2.28-2.85]per 100,000 population), and East Asia (2.42 [2.22-2.66] per 100,000 population) (Table 1, Fig. 1A). The Caribbean (5.5\% [- 7.15-20.22\%]) and South Asia (5.09\% [-3.13-14.68\%]) showed the largest increases 
from 2007 to 2017. In contrast, southern Sub-Saharan Africa had the greatest decrease in agestandardized incidence rate from 2007 to 2017 (-28.09\% [-35.61\% to $-18.91 \%])$, followed by western Europe (-17.96\% [-24.19\% to - 10.09\%]) and high-income Asia Pacific region (-17.52\% [-26.07\% to 7.53\%] ) (Table 1, Fig. 1D).

At the country level, global maps of the age-standardized NPC incidence rates in 2017 and the percentage change in them from 2007 to 2017 were presented in Table 2 and Fig. 2A and 2D.The highest agestandardized NPC incidence rate in 2017 was observed in Greenland (5.91 [4.98-7.07] per 100,000 population), followed by Malaysia (5.4 [4.06-7.05] per 100,000 population), and Guam (5.19 [3.99-6.6] per 100,000 population). In contrast, the lowest age-standardized rates in 2017 were found in Mozambique (0.16 [0.12-0.21] per 100,000 population), Montenegro (0.161 [0.13-0.19] per 100,000 population), and Chile (0.173 [0.13-0.22] per 100,000 population) (Table 2, Fig. 2A).

The percentage change in the age-standardized NPC incidence rates from 2007 to 2017 had a huge difference between nations, with Ukraine (37.95\% [8.03-77.53\%]), Jamaica (22.87\% [-14.08-70.66\%]), and Dominican Republic (14.33\% [-12.11-49.97\%]) showing the largest increases. In contrast, Iraq ($58.98 \%$ [ $-71.29 \%$ to $-39.75 \%]$ ), Israel (-37.93\% [ $-48.81 \%$ to $-24.27 \%]$ ), and Bahrain $(-36.04 \%$ [$51.39 \%$ to $-16.25 \%]$ ) showed the greatest decreases during this period (Table 2, Fig. 2D).

Figure 3A shows the NPC incidence rate in 2017 at the global level by SDI regions across different age groups from 15 to over 80 years. The NPC incidence rate increased in most regions from ages 15 to 54 years, reaching a peak in the 65-69-year age group in the high-SDI regions and in the 70-74-year age group in the low-middle-SDI regions. Globally, the high-middle-SDI and middle-SDI groups showed similar age-specific patterns:the NPC incidence rate increased up to 50-54 years, decreased until 55-59 years, and then increased again.

Figure 4A displays the global- and regional-level observed age-standardized NPC incidence rates from 2007 to 2017 and the prospective rates based on the SDI values. The age-standardized incidence rate in most regions decreases with the increase of SDI, especially in Southeast Asia.

\section{The DALY counts and age-standardized DALYs rate per 100,000 population by countries and territories}

Table 1 shows the number of DALYs for 2017, the age-standardized DALY rate per 100,000 population for 2017, the percentage change in all-age DALY counts, and the percentage change in age-standardized DALY rates in NPC between 2007 and 2017 by countries and territories. Globally, from 2007 to 2017, the number of DALYs increased to 2.09 [2-2.1] million (7), representing an increase of 18.4\% (13.99$23.09 \%)$. The age-standardized DALY rate was 25.45 (24.44-26.5) per 100,000 population in 2017, representing a decrease of $4.95 \%(-8.46 \%$ to $-1.24 \%)$ from 2007 to 2017 . The pattern of agestandardized DALY rates was also about the same between sexes, and these are summarized in Supplementary Tables 1 and 2. 
In 2017, among all seven super-regions, the highest age-standardized DALY rate of NPC was observed in the middle-SDI quintiles (32.95 [30.89-35.19] per 100,000 population) (Table 1, Fig. 1B). All super-regions showed a decreasing trend of age-standardized DALY rate,and the largest percentage decrease over the study period was observed in the high-SDI quintiles $(-14.85 \%$ [ $-17.94 \%$ to $-10.87 \%]$ (Table 1, Fig. $1 \mathrm{E})$.

In 2017, the geographic regions with the highest age-standardized DALY rates of NPC were Oceania with 64.14 [50.94-79.85] per 100,000 population, Southeast Asia with 49.6 [45.04-55.57] per 100,000 population, and East Asia with 41.39 [38.90-44.38] per 100,000 population (Table 1, Fig. 1B). From 2007 to 2017 , among the 21 analyzed regions, the Southern Sub-Saharan Africa $(-29.06 \%$ [ $-34.4 \%$ to $22.11 \%])$, Western Europe $(-18.52 \%$ [- $23.01 \%$ to $-12.7 \%])$, and Central Europe $(-18.32 \%$ [- $22.39 \%$ to $14.07 \%]$ ) showed the largest decreases. In contrast, the Caribbean(4.68\% [-3.37-13.17\%]) had the largest increase in the age-standardized DALY rate of NPC, followed by South Asia (3.34\% [- 2.789.96\%]) (Table 1, Fig. 1E).

Global maps of the age-standardized DALY rates of NPC in 2017 and the percentage change in the agestandardized DALY rates from 2007 to 2017 at the country level are presented in Table 2 and Fig. 2B and $2 \mathrm{E}$. The highest age-standardized DALY rate of NPC was observed in Greenland (123.81 [110.57-137.84] per 100,000 population), followed by Malaysia (99.34 [79.03-121.48] per 100,000 population), and Guam (98.74 [85.2-113.45] per 100,000 population). The lowest age-standardized rates in 2017 were observed in Chile (3.24 [2.82-3.72] per 100,000 population), Mozambique (3.84 [3.16-4.54] per 100,000 population), and Montenegro (3.97 [3.5-4.48] per 100,000 population (Table 2, Fig. 2B).

The percentage change in the age-standardized DALY rates of NPC from 2007 to 2017 also had a huge difference between nations, with Jamaica (23.4\% [- 0.82-49.19\%]), Ukraine (22.63\%[7.84-41.89\%]), and Dominica (14.04\% [1.71-27.58\%]) displaying the largest increases. In contrast, Iraq $(-61.51 \%[-68.15 \%$ to $-52.12 \%])$, Israel $(-38.64 \%$ [ $-45.49 \%$ to $-30.41 \%])$, and Bahrain $(-37.14 \%[-46.42-26.37 \%])$ showed the largest decreases during this period (Table 2, Fig. 2E).

Figures 3B shows the 2017 DALY rates of NPC at the global level by SDI regions across different age groups from 15 to over 80 years. The DALY rate increased in most regions from ages 15 to 59 years. In 2017, the DALY rate peaked in the 60-64-year age group globally.In the middle-SDI regions, the DALY rate peaked in the 65-69-year age.And in the high-SDI, low-middle-SDI, and low-SDI regions, the DALY rate peaked in the55-59-year age group. In the high-middle-SDI regions, the DALY rate increased up to 50-54 years, decreased until 55-59 years, and then increased again.

Figure 4B displays the global- and regional-level observed age-standardized NPC DALY rates from 2007 to 2017 and the prospective rates based on the SDI values. The age-standardized DALY rate in most regions decreases with the increase of SDI, especially in Southeast Asia.

\section{The death counts and age-standardized deaths rate per 100,000 population by countries and territories}


Table 1 also reveals the number of deaths in NPC for 2017, the age-standardized death rate per 100,000 for 2017, the percentage change in all-age death counts, and age-standardized death rates between 2007 and 2017 by countries and territories. Globally, from 2007 to 2017, the number of deaths of NPC increased to 69.55 [66.87-72.27] thousand, representing an increase of $24.39 \%$ [20.01-28.85\%] in global death counts in that period. The age-standardized death rate was 0.86 [0.82-0.89] per 100,000 population in 2017, representing a decrease of 3.01\% [-6.36-0.43\%] from 2007 to 2017.

In 2017, among all seven super-regions, the highest age-standardized death rate of NPC was found in the middle-SDI countries (1.14 [1.07-1.22] per 100,000 population) (Table 1, Fig. 1C). The largest percentage decrease in the age-standardized death rate from 2007 to 2017 was observed in the high-SDI countries ($13.29 \%[-16.34 \%$ to $-9.47 \%]$ ) (Table 1, Fig. 1F).

The geographic regions with the highest age-standardized death rates of NPC were Oceania with 2.06 [1.69-2.49] per 100,000, Southeast Asia with 1.73 (1.57-1.93) per 100,000, and East Asia with 1.42 [1.34-1.51] per 100,000 (Table 1, Fig. 1C). Among the 21 regions analyzed, southern Sub-Saharan Africa $(-25.59 \%[-30.51 \%$ to $-19.40 \%])$, central Europe $(-17.06 \%$ [ $-20.97 \%$ to $-13.10 \%])$, and western Europe $(-15.73 \%[-19.95 \%$ to $-10.26 \%])$ showed the largest decreases in age-standardized death rate of NPC from 2007 to 2017. In contrast, South Asia showed the highest increase (5.45\% [-0.59-12.15\%]), followed by the Caribbean (5.03\% [-3.05-13.64\%]) and Oceania (0.17\% [-10.27-12.14\%]) (Table 1, Fig. 1F).

Global maps of the age-standardized death rates of NPC in 2017 and the percentage change in them from 2007 to 2017 at the nation level are displayed in Table 2 and Fig. 2C and 2F. The highest agestandardized death rate of NPC was observed in Greenland (4.78 [4.29-5.3] per 100,000 population), followed by Malaysia (3.44 [2.79-4.2] per 100,000 population) and Guam (3.31 [2.89-3.75] per 100,000 population). The least age-standardized death rates in 2017 were observed in Chile $(0.112$ [0.099-0.129] per 100,000 population), Egypt (0.114 [0.092-0.138] per 100,000 population), and Iraq (0.12 [0.1070.135 ] per 100,000 population (Table 2, Fig. 2 C).

The percentage change in age-standardized death rates of NPC from 2007 to 2017 also varied greatly between countries, with Jamaica (20.93\% [-1.94-43.18\%]), Dominica (13.11\% [1.47-25.58\%]), and Dominican Republic (12.6\% [- 9.05-35.33\%]) displaying the largest increases. In contrast, Iraq (-63.3\% [ $-69.22 \%$ to $-55.62 \%])$, Israel $(-39.24 \%[-46 \%$ to $-31.15 \%])$, and Bahrain $(-38.68 \%[-47.34 \%$ to $28.55 \%]$ ) showed the largest decreases during this period (Table 2, Fig. 2F).

Figure 3C shows the NPC-specific death rate in 2017 at the global level by SDI regions across different age groups from 15 to over 80 years. In most regions,the death rate increased from ages 15 to 74 years. In 2017, the death rate peaked in the over-80-year age group in the high-SDI and middle-SDI groups. In the low- middle-SDI and low-SDI groups and globally, the NPC-specific death rate increased up to 70-74 years, declined until 75-79 years, and then increased again. 
Figure 4C dispiays the global- and regional-level observed age-standardized NPC death rates from 2007 to 2017 and the prospective rates based on the SDI values. The age-standardized death rate in most regions decreased with the increase of SDI, especially in Southeast Asia.

\section{The numbers of risk-attributable DALYs and death and the percentage changes in the risk-attributable age- standardized DALYs and death rates globally}

Table 3 reveals the numbers and age-standardized rate of risk-attributable DALYs and deaths of NPC in 2017 and the percentage changes in numbers and age-standardized rate of risk-attributable DALY and death for each cause. Globally, 1.22 million [1.09-1.36] DALYsand 41.67 thousand [37.06-46.16]deaths were ascribed to GBD-reported risk factors in 2017, which increased by $18.99 \%$ [13.34-25.73\%] and 23.5\% [17.76-29.84\%], from 2007 to 2017. Between 2007 and 2017, the age-standardized risk-

attributable DALY rate decreased to 14.83 [13.15-16.46] per 100,000 population, representing a decrease of $5.55 \%[-9.93-0.34 \%]$. Meanwhile, the age-standardized risk-attributable death rate decreased to 0.51 [0.45-0.57] per 100,000 population, representing a decrease of $4.25 \%(-8.64 \%$ to $-0.56 \%)$.

In 2017, occupational exposure to formaldehyde, smoking, alcohol use, and diet low in fruits were the primary four risk factors contributing to both DALYs and deaths of NPC. The first leading contributor to the age-standardized DALYs rate was alcohol use (11.57 [9.69-13.35]), representing a decrease of 3.59\% [-9.68-3.08\%] from 2007 to 2017 . This was followed by smoking (5.95 [4.23-7.57]), representing a decrease of $10.05 \%$ [ $-14.51 \%$ to $-4.9 \%]$, and then a diet low in fruits $(1.55$ [0-3.44]), representing a decrease of $13.48 \%$ [-19.31-712.17\%].

The highest risk-attributable age-standardized death rates were associated with alcohol use $(0.388$ [0.323-0.448]), representing a decrease of $2.24 \%(-8.35-4.26 \%)$ from 2007 to 2017 . This was followed by smoking $(0.223[0.162-0.28])$, representing a decrease of $8.17 \%$ [ $-12.54 \%$ to $-3.05 \%]$, and then a diet low in fruits $(0.053$ [0-0.118]), representing a decrease of $12.12 \%$ [-18.07-739.55\%].

\section{Discussion}

This report provides the latest available estimates of incidence of and DALYs and deaths associated with NPC in 194 countries and territories from 2007 to 2017. Globally, NPC accounted for10.978 million incident cases, 2.09 million DALYs, and 69.55 thousand deaths in 2017. Between 2007 and 2017, the agestandardized incidence rate decreased by $2.3 \%$ [-7-3.28\%], the age-standardized DALY rate decreased by $4.95 \%[-8.46 \%$ to $-1.24 \%]$, and the age-standardized death rate decreased by $3.01 \%[-6.36-0.43 \%]$.

Consistent with our findings, a global study also found decreasing trends in age-standardized rates of NPC incidence in southern and eastern Asia, North America, and the Nordic countries[3]. Nevertheless, absolute numbers of new NPC cases and NPC-attributable DALYs and deaths have increased as a result 
of population growth and aging. We observed that the age-standardized incidence, DALY, and death rates in 2017 were highest in the middle-SDI quintiles, whereas the largest percentage decrease in these rates were observed in the high-SDI quintiles, as NPC is a rare cancer in developed areas. The reason may be related to early prevention, screening, diagnosis and later treatment in developed areas.

The current standard NPC treatment incorporates radiation therapy combined with chemotherapy. However, due to the anatomical characteristics of the nasopharyngeal cavity and the biological characteristics of NPC, conventional local radiotherapy often involves important surrounding organs and tissues, which results in a series of side effects and sequelae[12]. Newly emerging treatments, such as immunology and targeted therapy[13-15], and the use of vaccines to prevent and treat EBV-infected potential NPC patients[16-17] (preventive vaccines and therapeutic vaccines) contributed to the decline of DALY and death rates.

Of the 21 regions analyzed, the highest age-standardized incidence in 2017, DALY, and death rates of NPC were observed in Oceania, Southeast Asia, and East Asia (at least double of those found in other areas). Furthermore, the Caribbean and South Asia showed the highest increases in the age-standardized incidence, DALY, and death rates of NPC from 2007 to 2017. NPC is endemic in southern China and Southeast Asia[18], and the decreases in China were probably due to the implementation of Epstein-Barr virus (EBV) screening for early diagnosis and prevention strategies, increased public awareness, and transform of eating habits[19-21]. The declines of DALY and death rates in some areas might partly due to a reduction in incidence rates. Improvements in diagnostic strategies and treatment methods were also main enabler[22-23].

We found that $77.42 \%$ of the new NPC cases were concentrated in Asian 2017. However, several high-risk countries in Asia, such as Indonesia, Malaysia, Philippines, Thailand, and China, showed a downward trend in age-standardized DALY rates and death rates of NPC, which may contribute to the overall global NPC burden. In addition, we observed the highest increase in the age-standardized incidence, DALY, and death rates of NPC in Ukraine, Jamaica, and Dominican Republic during 2007 to 2017. The exact reason for this observation requires further investigation in light of the actual situation in each country.

Currently, the etiology of NPC remains unclear, although there are clear epidemiological characteristics of regional aggregation and ethnic sensitivity. Compelling evidence suggests that genetic factors, EBV infection, environmental factors (e.g., premature exposure to chemical carcinogens, especially salted fish in southern China) are important etiological models of NPC[18]. In our study, occupational exposure to formaldehyde, smoking, alcohol use, and diet low in fruits were found to be the four main risk factors contributing to NPC both DALYs and deaths in 2017, suggesting that primary prevention should be focused on controlling and staying away from these risk factors. The risk-attributable age-standardized DALY and death rates of NPC both showed a significant decrease from 2007 to 2017, especially with respect to smoking ( $-10.05 \%$ and $-8.17 \%$, respectively); this may have benefited from the World Health Organization (WHO)'s introduction of the MPOWER policy, which was created to help implement the WHO Framework Convention on Tobacco Control so that significant progress can be made in global tobacco 
control. In order to further reduce the burden of NPC worldwide, the main implementation measures should focus on the control of NPC-related risk factors, such as prohibiting smoking in public places, reducing alcohol consumption, averting detrimental occupational exposures, and increasing the intake of fresh fruits and vegetables.

For head and neck cancer, the primary risk factors are smoking pack-years, alcoholism, and human papilloma virus infection for oropharyngeal cancer. As the cause of NPC is not yet clear, effective etiological prevention is impossible. The detection and tracking of multiple indicators for high-risk groups so as to achieve early detection and early diagnosis for secondary prevention is a feasible strategy. Notably, the early symptoms of NPC are similar to those of benign nasopharyngeal lesions, and early screening has not been widely popularized. Less than $20 \%$ of NPC patients are diagnosed early, and the vast majority of NPC patients are diagnosed in the middle and late stages[24]. EBV serology is the most important screening strategy, a study has revealed that EBV screening is cost-effective for NPC[3].

To our knowledge, this is the first study to provide a contemporary comprehensive assessment of the estimated NPC burden at the global, regional, and national levels by age, sex, and socio-demographic status in 194 countries and territories over time. We used the SDI to identify areas wherein the NPC burden is better or worse than expected. We also reported the DALYs, an intuitive comprehensive indicator that explains both the mortality and morbidity associated with a disease. These analyses enabled the contextualization of the disease burden via cross-disease and cross-geographic comparisons.

However, our research also has limitations. The calculations in GBD 2017 were based on an algorithm that used the currently available data in each country. As NPC is a rare disease, data in some regions might be limited; therefore, the Uls were wider in areas where fewer data points were available.

In conclusion, from 2007 to 2017, the global burden of NPC has decreased continuously, especially in the middle-SDI quintiles, Southeast Asia, East Asia,and various other countries. The decreased burdens of risk-attributable DALY and death rates were mainly attributable to changes in alcohol use, smoking, and consumption of diet low in fruits. Interventions should be targeted at the established high-risk populations and regions.

\section{Abbreviations}

DALYs: Disability-Adjusted Life Years; NPC: Nasopharyngeal Carcinoma; GHDx: Global Health Data Exchange. GBD: Global Burden of Diseases; SDI: Socio-Demographic Index; GATHER: Guidelines for Accurate and Transparent Health Estimates Reporting; YLLs: years of life lost; YLDs: years lived with disability; Ul: uncertainty interval;

\section{Declarations}

\section{Acknowledgments}


We would like to thank the anonymous reviewers for their insightful comments and valuable suggestions.

\section{Author contributions}

ZSS and LYH conceived the idea for this initiative. JL and ZSS contributed to reading the literature, preparation of figures and the table, and writing the manuscript; LYH, SSG, LQW, YQL and HXD assisted with writing and revised the manuscript. All authors read and approved the final manuscript.

\section{Funding}

This study was supported by Ningbo medical and health brand discipline(No.PPXK2018-02);Zhejiang Provincial Natural Science Foundation of China(LY19H160014;LQ21H130001); Ningbo "Technology Innovation 2025" Major Special Project (2020Z097); Medical and Health Research Project of Zhejiang Province(2019ZD018;2021KY307); Ningbo Natural Science Foundation(202003N4239).

\section{Availability of data and materials}

The burden of NPC data are publically available from the Global Health Data Exchange (GHDx) query tool (http://ghdx.healthdata.org/gbd-results-tool).

\section{Ethics approval and consent to participate}

Not applicable.

\section{Consent for publication}

Not applicable.

\section{Competing interests}

The authors declare that they have no competing interests.

\section{References}

1. Limkin EJ, Blanchard P. Does East meet West? Towards a unified vision of the management of Nasopharyngeal carcinoma. Br J Radiol. 2019;92(1102):20190068.

2. Tsang CM, Lui VWY, Bruce JP, Pugh TJ, Lo KW. Translational genomics of nasopharyngeal cancer. Semin Cancer Biol. 2020;61:84-100.

3. Tang LL, Chen WQ, Xue WQ, He YQ, Zheng RS, Zeng YX, et al. Global trends in incidence and mortality of nasopharyngeal carcinoma. Cancer Lett. 2016;374(1):22-30.

4. GBD 2017 Causes of Death Collaborators. Global, regional, and national age-sex-specific mortality for 282 causes of death in 195 countries and territories, 1980-2017: a systematic analysis for the Global Burden of Disease Study 2017. Lancet. 2018;392(10159):1736-88. 
5. GBD 2017 Disease and Injury Incidence and Prevalence Collaborators. Global, regional, and national incidence, prevalence, and years lived with disability for 354 diseases and injuries for 195 countries and territories, 1990-2017: a systematic analysis for the Global Burden of Disease Study 2017. Lancet. 2018;392(10159):1789-858.

6. GBD 2017 DALYs and HALE Collaborators. Global, regional, and national disability-adjusted life-years (DALYs) for 359 diseases and injuries and healthy life expectancy (HALE) for 195 countries and territories, 1990-2017: a systematic analysis for the Global Burden of Disease Study 2017. Lancet. 2018;392(10159):1859-922.

7. GBD 2017 Risk Factor Collaborators. Global, regional, and national comparative risk assessment of 84 behavioural, environmental and occupational, and metabolic risks or clusters of risks for 195 countries and territories, 1990-2017: a systematic analysis for the Global Burden of Disease Study 2017. Lancet. 2018;392(10159):1923-94.

8. Stevens GA, Alkema L, Black RE, Boerma JT, Collins GS, Ezzati M, et al. Guidelines for Accurate and Transparent Health Estimates Reporting: the GATHER statement. Lancet. 2016;388(10062):e19-e23.

9. GBD 2017 Population and Fertility Collaborators. Population and fertility by age and sex for 195 countries and territories, 1950-2017: a systematic analysis for the Global Burden of Disease Study 2017. Lancet. 2018;392(10159):1995-2051.

10. GBD 2016 Risk Factors Collaborators. Global, regional, and national comparative risk assessment of 84 behavioural, environmental and occupational, and metabolic risks or clusters of risks, 1990-2016: a systematic analysis for the Global Burden of Disease Study 2016. Lancet. 2017;390(10100):1345422.

11. El Bcheraoui C, Mimche H, Miangotar Y, Krish VS, Ziegeweid F, Krohn KJ, et al. Burden of disease in francophone Africa, 1990-2017: a systematic analysis for the Global Burden of Disease Study 2017. Lancet Glob Health. 2020;8(3):e341-e351.

12. Tuan JK, Ha TC, Ong WS, Siow TR, Tham IW, Yap SP, et al. Late toxicities after conventional radiation therapy alone for nasopharyngeal carcinoma. Radiother Oncol. 2012;104(3):305-11.

13. Louis CU, Straathof K, Bollard CM, Ennamuri S, Gerken C, Lopez TT, et al. Adoptive transfer of EBVspecific $T$ cells results in sustained clinical responses in patients with locoregional nasopharyngeal carcinoma. J Immunother. 2010;33(9):983-90.

14. Wildeman MA, Novalic Z, Verkuijlen SA, Juwana H, Huitema AD, Tan IB, et al. Cytolytic virus activation therapy for Epstein-Barr virus-driven tumors. Clin Cancer Res. 2012;18(18):5061-70.

15. Ma BBY, Hui EP, Chan ATC. Investigational drugs for nasopharyngeal carcinoma. Expert Opin Investig Drugs. 2017;26(6):677-85.

16. Hui EP, Taylor GS, Jia H, Ma BB, Chan SL, Ho R, et al. Phase I trial of recombinant modified vaccinia ankara encoding Epstein-Barr viral tumor antigens in nasopharyngeal carcinoma patients. Cancer Res. 2013;73(6):1676-88.

17. Taylor GS, Jia H, Harrington K, Lee LW, Turner J, Ladell K, et al. A recombinant modified vaccinia ankara vaccine encoding Epstein-Barr Virus (EBV) target antigens: a phase I trial in UK patients with 
EBV-positive cancer. Clin Cancer Res. 2014;20(19):5009-22.

18. Chang ET, Adami HO. The enigmatic epidemiology of nasopharyngeal carcinoma. Cancer Epidemiol Biomarkers Prev. 2006;15(10):1765-77.

19. Cao SM, Simons MJ, Qian CN. The prevalence and prevention of nasopharyngeal carcinoma in China. Chin J Cancer. 2011;30(2):114-9.

20. Lau HY, Leung CM, Chan YH, Lee AW, Kwong DL, Lung ML, et al. Secular trends of salted fish consumption and nasopharyngeal carcinoma: a multi-jurisdiction ecological study in 8 regions from 3 continents. BMC Cancer. 2013;13:298.

21. Jia WH, Luo XY, Feng BJ, Ruan HL, Bei JX, Liu WS, et al. Traditional Cantonese diet and nasopharyngeal carcinoma risk: a large-scale case-control study in Guangdong, China. BMC Cancer. 2010;10:446.

22. Lee AW, Foo W, Mang O, Sze WM, Chappell R, Lau WH, et al. Changing epidemiology of nasopharyngeal carcinoma in Hong Kong over a 20-year period (1980-99): an encouraging reduction in both incidence and mortality. Int J Cancer. 2003;103(5):680-5.

23. Zhang LF, Li YH, Xie SH, Ling W, Chen SH, Liu Q, et al. Incidence trend of nasopharyngeal carcinoma from 1987 to 2011 in Sihui County, Guangdong Province, South China: an age-period-cohort analysis. Chin J Cancer. 2015;34(8):350-57.

24. Yang S, Wu S, Zhou J, Chen XY. Screening for nasopharyngeal cancer. Cochrane Database Syst Rev. 2015;2015(11):Cd008423.

\section{Tables}

Due to technical limitations, tables PDF is only available as a download in the Supplemental Files section.

\section{Figures}


A

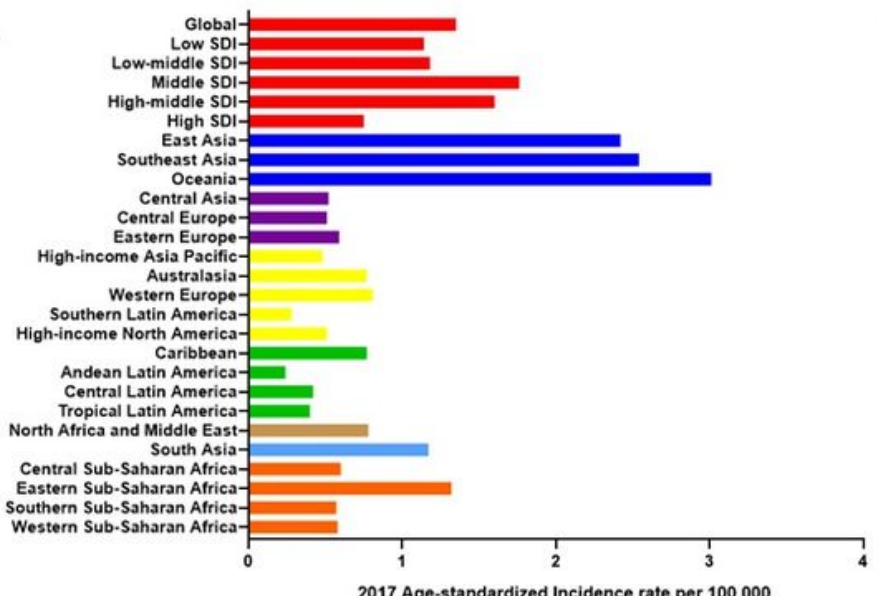

B

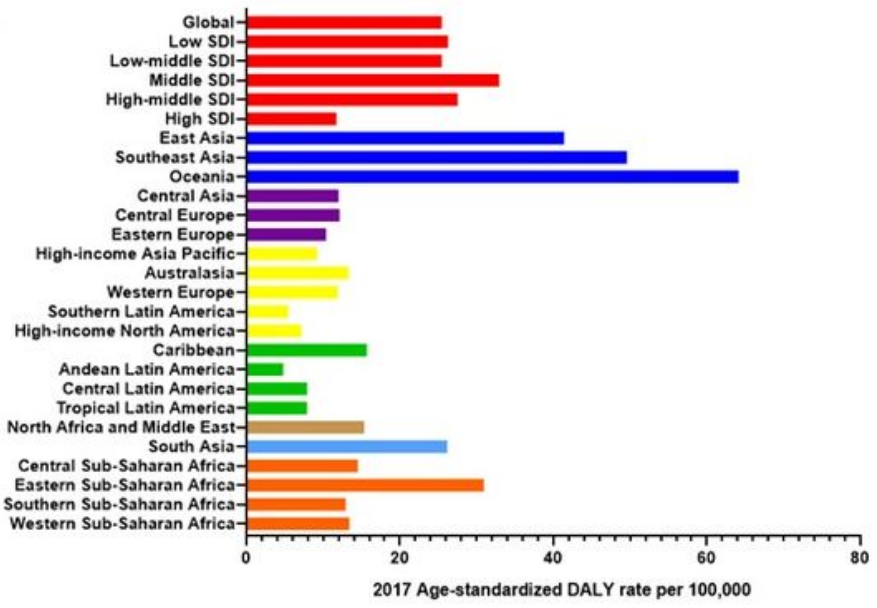

C

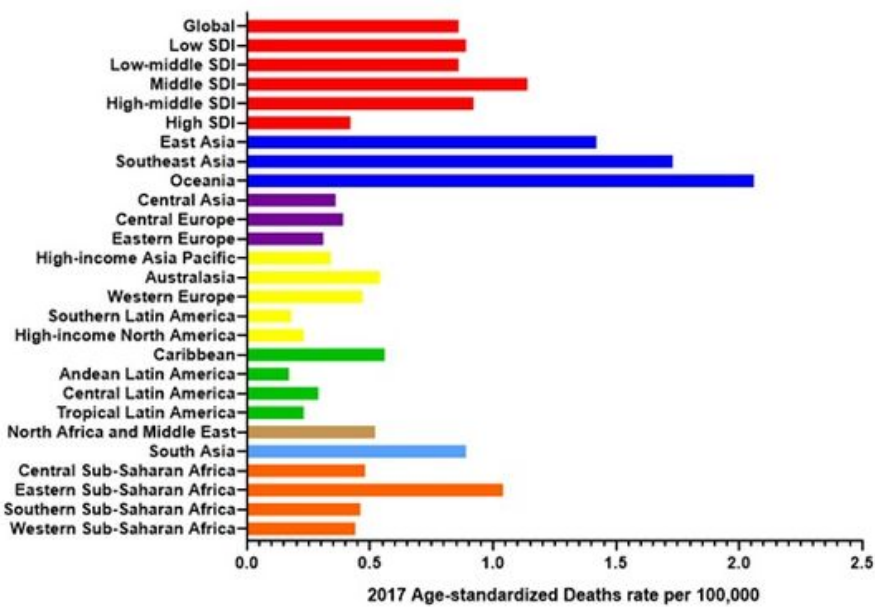

D

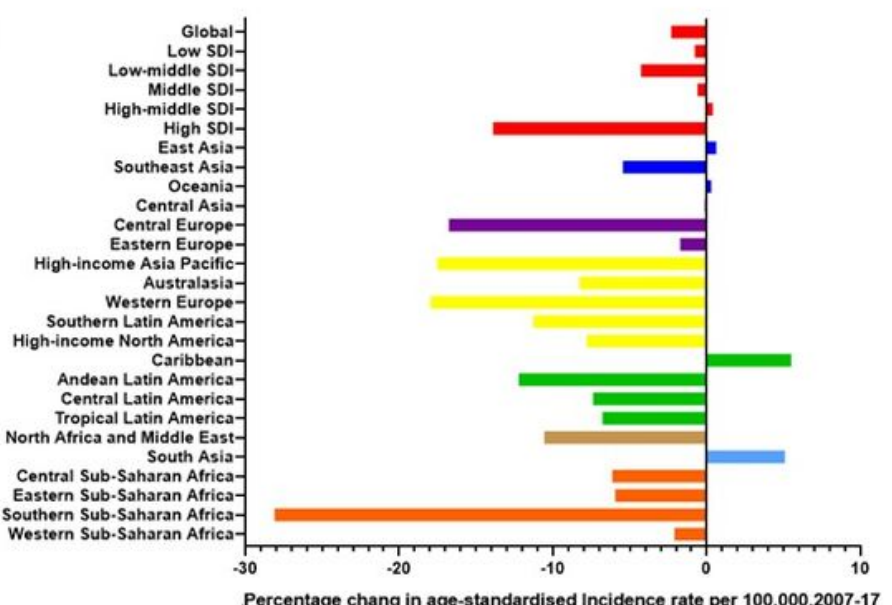

E

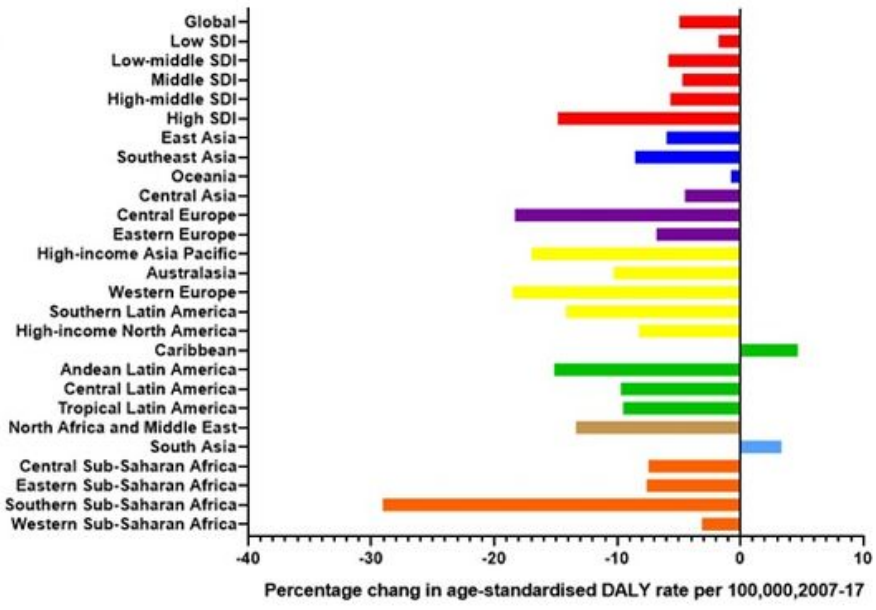

F

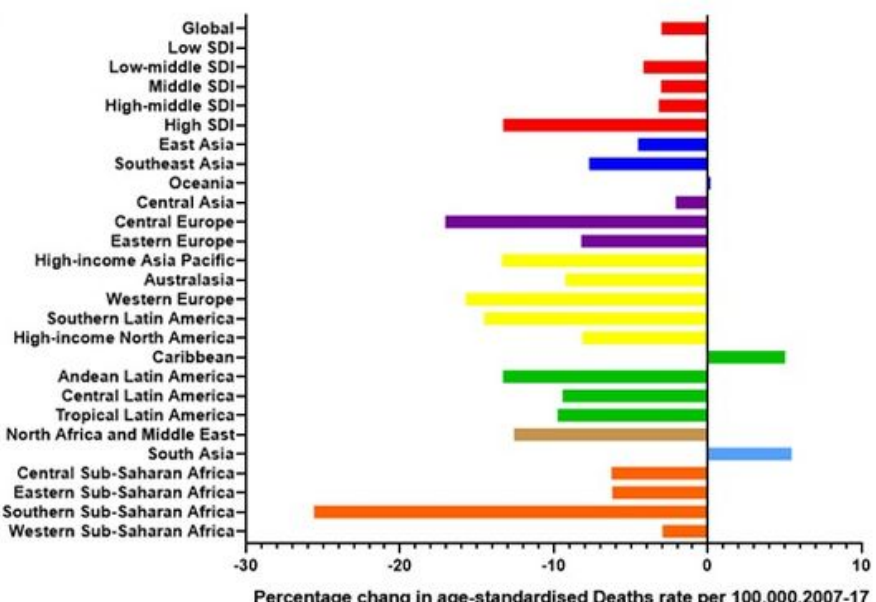

\section{Figure 1}

Age-standardized rate in 2017 and percentage change in age-standardized rate by SDI and world regions, 2007-17. Age-standardized rate in 2017 changes due to Incidence (A), DALYs (B), Deaths (C); Percentage change in age-standardised rate changes in Incidence (D), DALYs (E), and Deaths (F). Both sexes. 


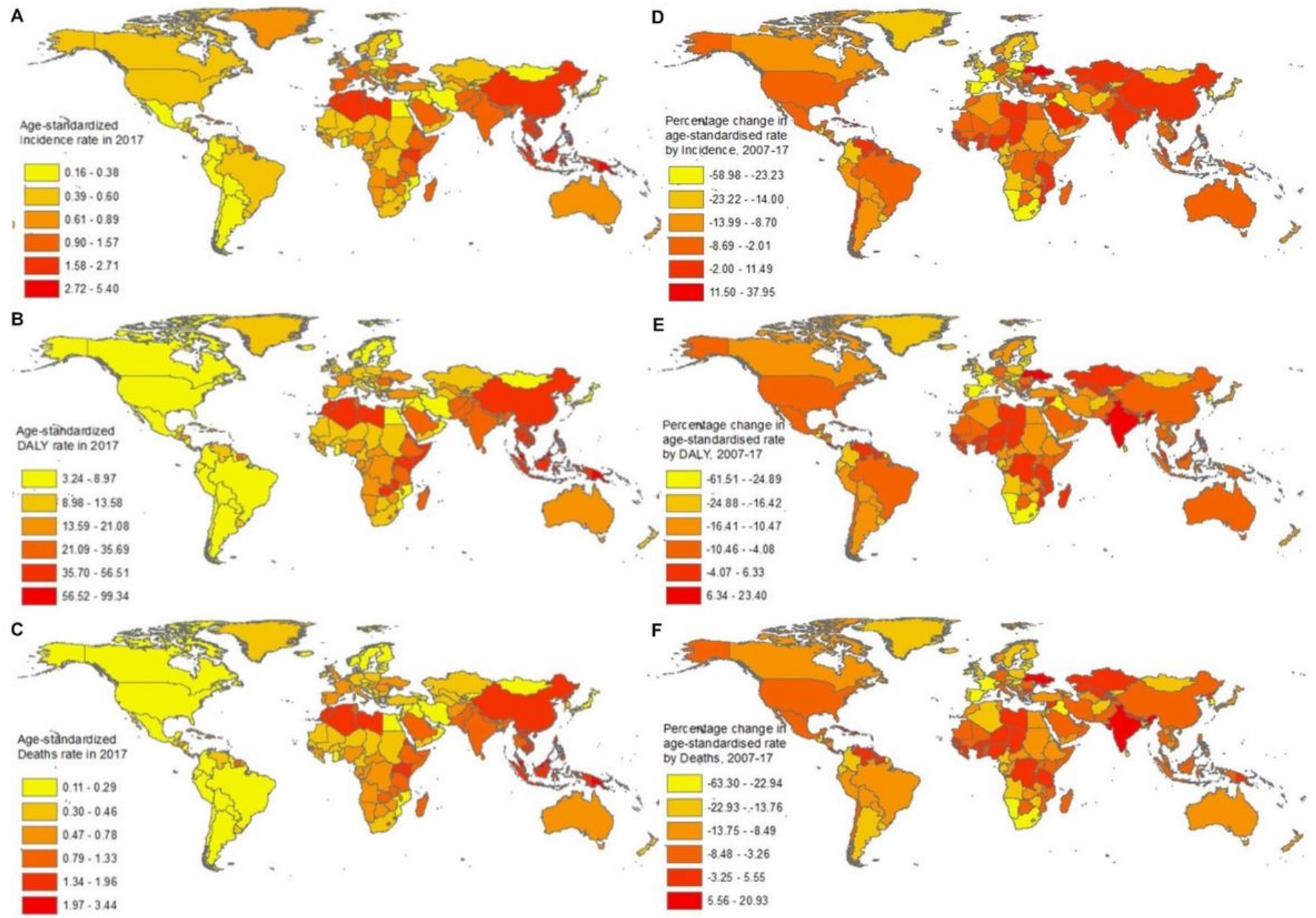

Figure 2

Age-standardized rate in 2017 and percentage change in age-standardized rate by location, 2007-17. Age-standardized rate changes due to Incidence (A), DALYs (B), Deaths(C); Percentage change in agestandardised rate changes in Incidence (D), DALYs (E), Deaths(F). Both sexes. 
A

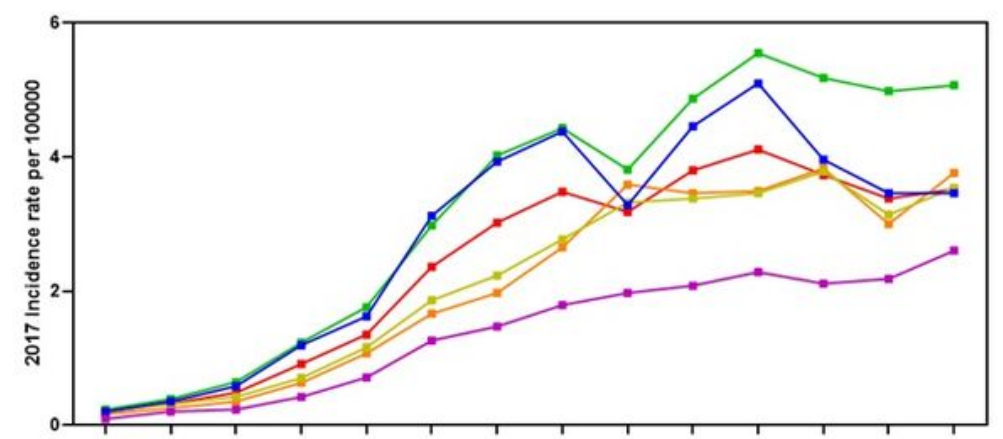

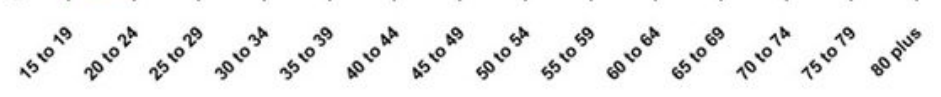

- Global $\rightarrow$ Low SDI $=$ Low-middle SDI $\rightarrow$ Middle SDI $\rightarrow$ High-middle SDI $\rightarrow$ High SDI

B

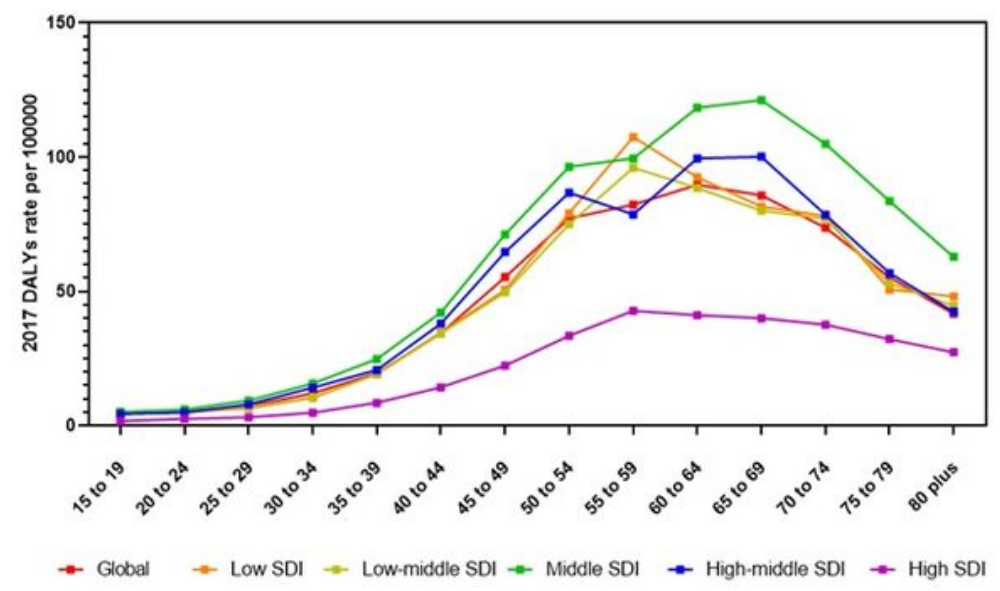

C

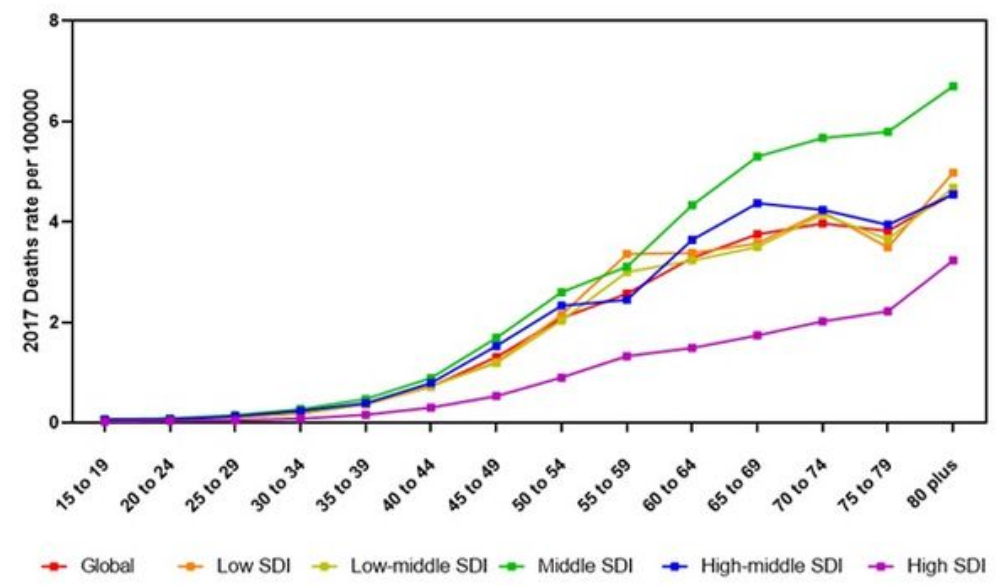

Figure 3

Age-SDI-specific Incidence, DALYs and Deaths rate per 100000 population ofNPC in 2017. Both sexes. 
A

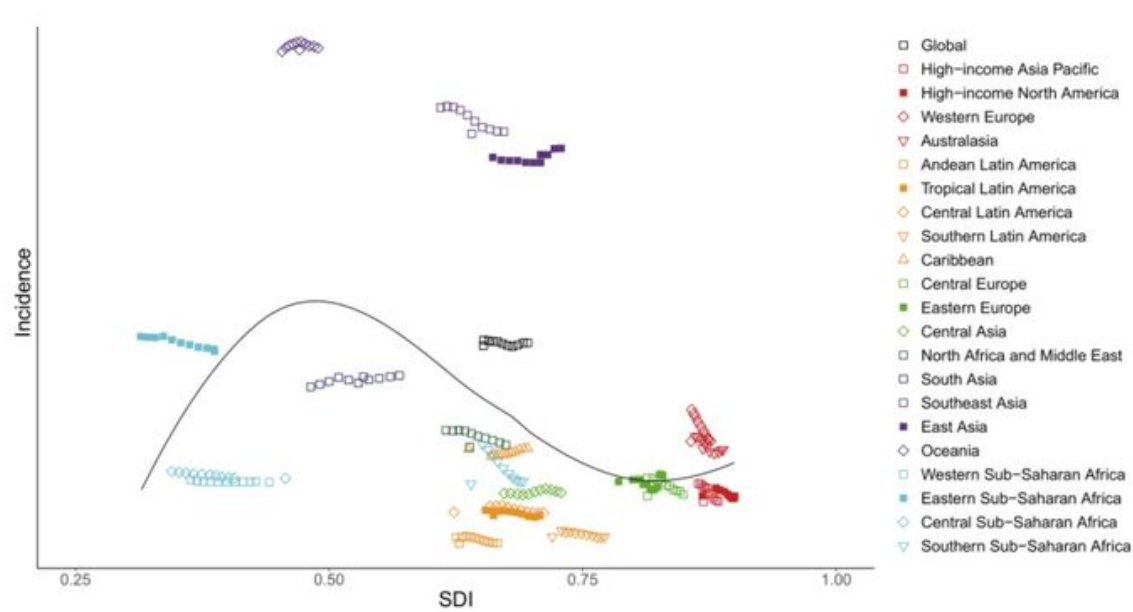

B

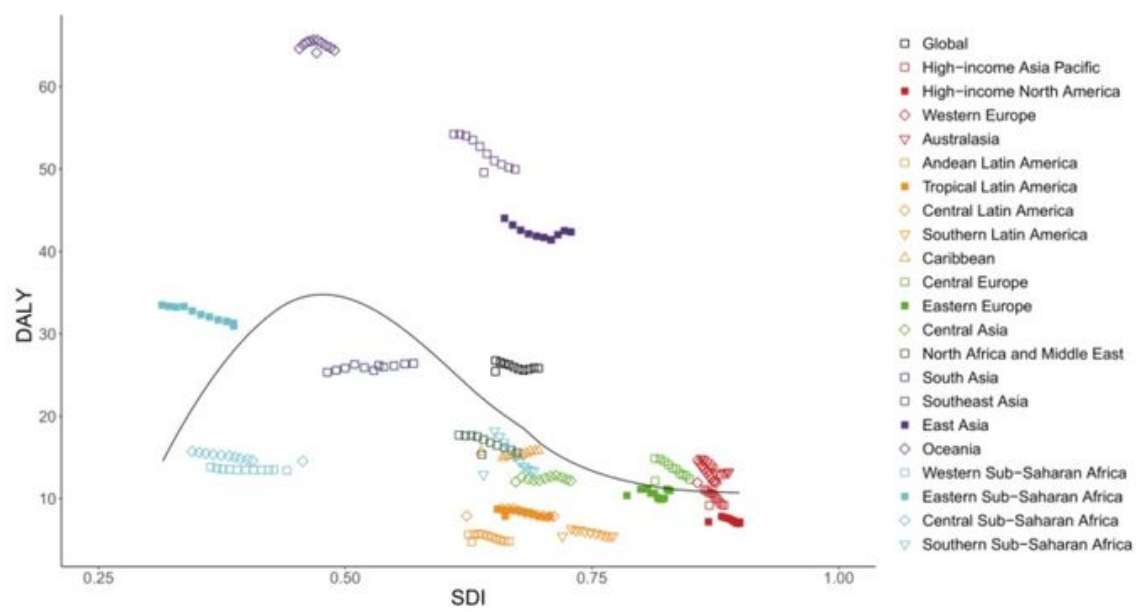

C

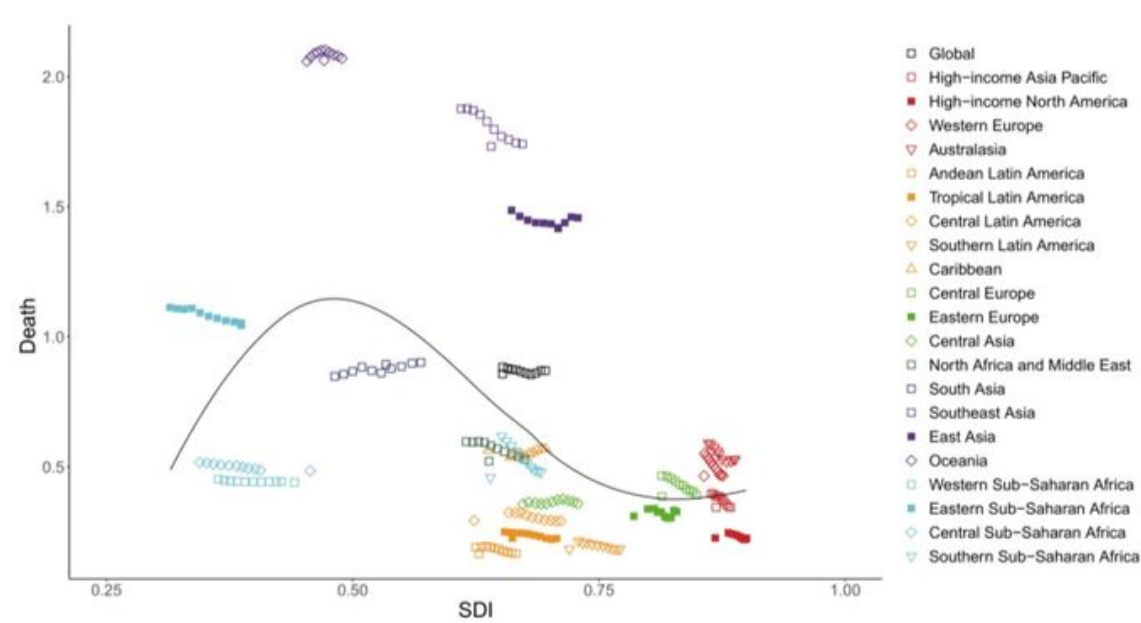

Figure 4

Age-standardized Incidence, DALYs and Deaths ratefor NPC by SDI, 2007-2017. Both sexes.

\section{Supplementary Files}

This is a list of supplementary files associated with this preprint. Click to download. 
- Supplementarytables16.pdf

- Tables13.pdf 